Revue Interventions économiques

Papers in Political Economy

$55 \mid 2016$

D'un régionalisme à l'autre : intégration ou interconnexion?

\title{
Les puissances émergentes dans la bataille mondiale de l'attraction : Bollywood, vecteur du soft power de l'Inde?
}

Emerging Powers in the Global Battle of Attraction: Bollywood, Vector of India's

Soft Power?

\section{Antonios Vlassis}

\section{OpenEdition}

\section{Journals}

Édition électronique

URL : http://journals.openedition.org/interventionseconomiques/2867

DOI : 10.4000/interventionseconomiques.2867

ISBN : $1710-7377$

ISSN : $1710-7377$

Éditeur

Association d'Économie Politique

Référence électronique

Antonios Vlassis, « Les puissances émergentes dans la bataille mondiale de l'attraction : Bollywood, vecteur du soft power de l'Inde? », Revue Interventions économiques [En ligne], 55 | 2016, mis en ligne le 29 juin 2016, consulté le 10 décembre 2020. URL : http://journals.openedition.org/

interventionseconomiques/2867; DOI : https://doi.org/10.4000/interventionseconomiques.2867

Ce document a été généré automatiquement le 10 décembre 2020.

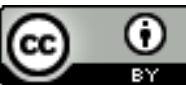

Les contenus de la revue Interventions économiques sont mis à disposition selon les termes de la Licence Creative Commons Attribution 4.0 International. 


\section{Les puissances émergentes dans la bataille mondiale de l'attraction : Bollywood, vecteur du soft power de l'Inde?}

Emerging Powers in the Global Battle of Attraction: Bollywood, Vector of India's

Soft Power?

Antonios Vlassis

1 En 2011, l'ancien premier ministre de l'Inde, Manmohan Singh, déclare que « le soft power indien est un élément de plus en plus important pour promouvoir notre empreinte globale. La richesse de la tradition indienne et la vitalité de la culture indienne contemporaine font des vagues dans le monde entier " (Thussu, 2013 : 127-128). De son côté, en 2009, Shashi Tharoor, ancien ministre des Affaires étrangères du gouvernement Singh, affirme que "l'Inde devient une superpuissance grâce à son soft power, sa capacité ( ability) de partager sa culture à travers le monde grâce à sa musique, sa technologie et Bollywood» (TEDIndia, 2009).

2 L'essor économique formidable de l'Inde et l'influence qu'elle tend à exercer désormais sur les affaires internationales conduisent les analystes à s'interroger sur son statut et son positionnement international. L'Inde est-elle une puissance émergente en quête du statut d'une puissance majeure ? Est-elle une puissance régionale qui ambitionne de devenir une puissance mondiale ? (Brewter, 2012 ; Dupont, 2009 ; Jain, 2008 ; Kapur, 2006 ; Nayar, 2003 ; Panagariya, 2008). Pour répondre à ces questions, l'analyse du soft power indien est centrale. Il a récemment fait l'objet de nombreuses études en provenance de deux disciplines scientifiques.

D'un côté, plusieurs analyses issues de travaux sur la communication et les médias voient dans Bollywood une partie de la puissance douce de l'Inde et un instrument du rayonnement international du pays (Thussu, 2013 ; Schaefer et Karan, 2013) et elles constatent que le cinéma populaire indien a acquis une dimension internationale que seul 
Hollywood peut concurrencer (Thussu, 2012). À cet égard, il s'agit d'illustrer autant un paysage cinématographique bipolaire fondé sur la concurrence de deux pôles de puissance, Hollywood et Bollywood (Augros, 2011 ; Matusitz, 2012 ; Tyrrell, 1999) que l'avènement d'un grand nombre de pôles de production culturelle remettant en cause l'hégémonie occidentale, voire américaine dans le contexte d'une globalisation de plus en plus poussée (Tunstall, 2007).

D'un autre côté, des études sous le prisme des Relations Internationales (RI) se concentrent sur l'action culturelle extérieure de l'Inde et son soft power pour mettre l'accent sur l'influence internationale du pays. Elles incluent dans leur propre définition de la puissance douce un grand nombre de domaines hétéroclites tels que le commerce, les valeurs au sens large du terme, la religion, l'éducation (Jaffrelot, 2008 ; Hymans, 2009 ; Kugiel, 2012; Wagner, 2010). Néanmoins, malgré leur importance économique et symbolique et leur expansion actuelle ${ }^{1}$, le poids des industries culturelles, notamment celui du cinéma, reste encore un facteur méconnu pour comprendre le rôle que l'Inde peut et veut jouer dans le monde.

Rappelons d'ailleurs que l'analyse des ressources intangibles de la puissance fait notamment irruption dans la discipline des RI lors du débat des années 1980 sur l'éventuel déclin de l'hégémonie américaine (Battistella, 2013 : 239)². En 1990, Joseph Nye (1990, 2004) développe le concept de 'soft power' pour mettre en lumière les métamorphoses actuelles des composantes de la puissance d'un État ${ }^{3}$. Pour le politologue américain, si les États-Unis désirent être la puissance hégémonique du $21^{\mathrm{e}}$ siècle, ils doivent renforcer leur soft power, fondé sur des ressources intangibles, telles que l'information, la culture, l'idéologie et les institutions. En 1988, inscrite dans la perspective hétérodoxe de l'économie politique internationale remettant en cause une conception exclusivement relationnelle de la puissance (Paquin, 2013 : 261-298), Susan Strange élabore, quant à elle, le concept de 'puissance structurelle' pour mettre l'accent sur les capacités matérielles et immatérielles des États-Unis en vue de déterminer les structures de la scène internationale (structure de sécurité, structure de production, structure financière, structure des savoirs) et exercer un leadership à l'échelle mondiale (Strange, 1994). Alors que ces deux auteurs se sont penchés sur des variables multiples pour analyser les transformations de la puissance à l'ère de la mondialisation, les industries culturelles ont occupé une place marginale dans leur réflexion. Toutefois, les travaux de Susan Strange ont récemment inspiré des analyses sur le capitalisme culturel des États-Unis, dont les vecteurs demeurent les firmes multinationales d'entertainment, basées essentiellement aux majors hollywoodiennes (Scott, 2004 ; Laroche et Bohas, 2005 ; Bohas, 2010). Comme le souligne Josepha Laroche, «l'interétatique et le transnational se trouvent indissociablement liés; la diplomatie du gouvernement des États-Unis et celle de la Motion Pictures Association s'enchevêtrent pour assurer la suprématie d'Hollywood sur le marché mondial des biens cinématographiques » (Laroche, $2013: 644$ ). Pour sa part, Peter J. Katzenstein (2005: 149-178) a consacré une partie importante de ses recherches récentes aux industries culturelles et à leur poids symbolique pour saisir la configuration de puissance parmi les entités régionales de la scène internationale.

6 Art industriel, art collectif, art de masse, art de la modernité, le cinéma est «un instrument du soft power des nations " (Dagnaud, 2011). Même si l'action culturelle extérieure d'un pays vise des objectifs si diffus et à si long terme qu'il est difficile d'évaluer l'étendue de leur impact (Morin, 2013 : 41), il est nécessaire de s'interroger sur le positionnement de l'Inde au sein du paysage cinématographique mondial et de savoir 
dans quelle mesure le cinéma permet à l'Inde de construire un statut de puissance active pourvue d'une capacité de façonner les structures de son environnement international (Santander, $2013: 524$ ). Pour saisir ces questions, notre analyse s'articule en trois temps : il s'agit d'abord d'analyser l'évolution des liens entre les autorités et l'industrie cinématographique indiennes et les structures institutionnelles du système cinématographique indien, avant de faire un état des lieux du rôle de l'Inde au sein du régime international de l'audiovisuel. On se penchera enfin sur la concurrence culturelle mondiale à laquelle l'Inde est confrontée et notamment sur son positionnement vis-à-vis des majors hollywoodiennes qui semblent être les acteurs-clés du paysage cinématographique mondial.

\section{Inde et cinéma : entre diversité régionale et autosuffisance}

7 L'essor du cinéma indien démarre très tôt dans les années 1920 lorsque ce pays est encore sous tutelle britannique. Comme le rappelle Joël Farges (2006: 691), « l'Inde avait reconnu dans cette nouvelle invention un spectacle qui lui venait de sa propre culture (...) il n'existe pas par le monde, de pays qui ait aussi vite et aussi totalement intégré une invention étrangère ».

\subsection{Intervention étatique discrète}

8 La première véritable intervention du gouvernement indien a eu lieu dans les années 1960 : la création de la Film Finance Corporation, chargée de financer des films et celle de l'Institut du film de Pune, école nationale du cinéma en 1960, ainsi que la mise en place de la Film and Television Institute of India en 1964, équivalent d'une Cinémathèque. En 1980, la Film Finance Corporation a intégré l'Indian Motion Picture Export Corporation - chargée de l'importation des films étrangers et de l'exportation du film indien - et elle est devenue la National Film Development Corporation (NFDC) (Deprez, 2010 : 37-44). Attachée au ministère indien d'Information et d'Audiovisuel (Ministry of Information and Broadcasting), cette dernièreconstitue la seule instance étatique d'aide à la production et à la diffusion des films en Inde, mais aussi à l'exportation des films indiens, à l'importation des films étrangers, aux coproductions internationales et aux projets techniques. Néanmoins, sa capacité financière et règlementaire est fort restreinte: jusqu'à présent, la NFDC a financé au total environ 300 films (surtout des films d'auteur), nombre trop limité compte tenu d'une production annuelle indienne de plus de 1000 films. Durant la période 2011-2012, son budget affiche une croissance de $38 \%$ et atteint environ 35 millions d'euros (voir NFDC, 2014), somme modeste comparée au budget du Centre national du Cinéma en France qui s'élève à 806 millions d'euros en 2011. À cet égard, l'intervention de la NFDC ne couvre qu'une part infime de la production cinématographique nationale et le manque de moyens et de volonté politique n'autorisent pas à parler d'une politique interventionniste à l'échelle gouvernementale, même dans les années 1960 et 1970 où l'économie indienne reposait sur un capitalisme d'État ${ }^{4}$.

9 Pour ce qui concerne les politiques règlementaires, l'adoption par le gouvernement indien de mesures telles que des quotas de projection (Mingant, $2010: 40-41$ ), un contrôle des investissements, des déductions fiscales, un partage des recettes, est largement sporadique (Pendakur, 1996 ; Mukharjee, 2002; Mukharjee, 2005). Il s'avère que la 
panoplie des mesures publiques prises par le gouvernement indien correspond à un protectionnisme très discret. Les aides financières et règlementaires en faveur du cinéma sont beaucoup plus restreintes que dans d'autres pays asiatiques pourvus d'une industrie cinématographique dynamique, tels que la Chine et la Corée du Sud. En d'autres termes, "l'Inde a développé pratiquement sans l'aide de l'État une industrie du divertissement de grande ampleur » (Dagnaud, 2009: 3).

\subsection{Préserver la diversité régionale}

Dans la mesure où le soft power en Inde n'est pas fortement associé à une politique de l'État, il est important de mettre en lumière les dynamiques territoriales qui marquent le paysage cinématographique du pays. En Inde, nous retrouvons un système de taxation privilégiant les films régionaux dans les différentes provinces du pays. Les films provenant d'une autre région ou de l'étranger sont soumis à des taxes plus élevées. Ainsi, le Bengale prélève-t-il $41 \%$ des recettes sur les entrées réalisées par des films externes, contre $16 \%$ pour la production bengalie. Au Karnataka, l'un des quatre États du sud de l'Inde, les films extérieurs doivent verser une taxe s'élevant à $52 \%$ des recettes, alors qu'aucun impôt n'est levé sur les films en kannada, langue officielle de la région.

D'ailleurs, Bollywood ${ }^{5}$, utilisé à l'étranger pour designer l'industrie cinématographique indienne, est le surnom donné au cinéma de langue hindi. Pour autant, il ne représente pas plus du quart de la production totale indienne annuelle. «La production cinématographique indienne est généralement associée à Bollywood et cache une réalité beaucoup plus complexe» (Deprez, 2011: 114). Il existe, en effet, d'autres industries régionales telles que Kollywood qui désigne le cinéma tamoul dans la région de Tamil Nadu et Tollywood pour le cinéma de langue télougou dans la région d'Andhra Pradesh. En 2011, la production cinématographique indienne atteint 1255 films en 24 langues différentes, dont 206 films en hindi, 192 en télougu, 185 en tamoul et seulement six en anglais (Central Board of Film Certification, 2011). Au total, les quatre États du sud de l'Inde, à savoir Tamil Nadu, Andhra Pradesh, Karnataka et Kerala, représentent le plus important producteur cinématographique du pays (Velayutham, $2008: 1$ ). Il s'avère que le marché cinématographique indien est moins un marché national monolithique qu'une mosaïque régionale composée de plusieurs pôles de production ${ }^{6}$ et de distribution, favorisant une diversité linguistique (Chitrapu, 2013).

\subsection{Un marché domestique dominé par les films indiens}

12 Une caractéristique du paysage cinématographique indien est son langage filmique singulier, à savoir le film 'masala' qui domine depuis les années 1970 le cinéma national. Celui-ci est un film de divertissement mélangeant amour, humour, action, drame, danse, musique et se terminant par un happy-end conventionnel, sans fonction d'être un révélateur ou dénonciateur des injustices sociales, des confrontations religieuses ou des inégalités économiques. "Ce cinéma ne s'intéresse pas directement aux convulsions sanglantes qui secouent le pays » (Farges, $2000: 164)^{7}$. Une grande partie de la production prolifique adopte ce langage filmique spécifique, adapté au goût du public.

13 En outre, durant la période 2005-2011, la production moyenne de films en Inde s'élève à 1203, beaucoup plus que celle des États-Unis (757), de la Chine (432), du Japon (414), de la Russie (292) ou de la France (239) (UNESCO Institute for Statistics, 2013 : 14). La machine 
productive privée en Inde (producteurs, distributeurs et organismes financiers) aboutit à saturer les écrans de productions nationales. En salle, les films étrangers représentent à peine $10 \%$ de la fréquentation totale. « La filière cinématographique indienne s'émancipe très tôt de sa dépendance vis-à-vis de l'étranger, et surtout d'Hollywood, pour fonctionner selon son propre modèle » (Deprez, 2011 : 116). À la différence d'autres pays asiatiques, tels que la Chine, la Corée du Sud et le Japon, les films indiens dominent entièrement le paysage cinématographique du pays (Table I). Les tentatives des majors hollywoodiennes de pénétrer le marché cinématographique intérieur n'ont pour le moment que peu de résultats malgré la politique de libéralisation du pays engagée depuis le début des années 1990 qu'aucun des gouvernements ne remettra en cause par la suite (Jaffrelot, $2006: 15)$. À cela s'ajoute que toutes les sociétés majeures de production et de distribution du marché cinématographique domestique restent indiennes sauf une exception notable: Fox Star Studios, un partenariat commercial entre la major hollywoodienne 20th Century Fox et la société indienne STAR, toutes les deux faisant partie du groupe de communication News Corporation.

Enfin, l'industrie cinématographique indienne se caractérise par une production en série nécessitant des ressources financières peu élevées. En 2006, « le budget moyen d'un film indien est 0,1 million USD, alors que le budget moyen d'un film américain atteint 30,7 millions, celui d'un film britannique 11,6 millions, et celui d'un film français 7,1 millions » (Chitrapu, 2013 : 18). Récemment, des grandes sociétés indiennes, telles que Yash Raj Films, Sun Pictures, Red Chillies Entertainment, Dharma, entendent investir des sommes considérables à la production et la distribution des films ${ }^{8}$. Durant la période 2013-2015, cinq productions indiennes ${ }^{9}$ se sont dotées d'un budget supérieur à 20 millions USD. Cependant, ces montants restent largement moins élevés que ceux de grosses productions hollywoodiennes dont le budget moyen est estimé à plus de 120 millions USD.

À cet égard, ces particularités du paysage cinématographique indien expliquent en grande partie la domination des films indiens dans le marché domestique, mais aussi la faiblesse du cinéma indien de s'exporter.

\subsection{Le marché intérieur, la frontière du cinéma indien}

16 Le marché des films indiens hors du pays reste encore très limité. D’un côté, des films indiens sont distribués dans des pays pourvus des communautés indiennes de la diaspora, tels que le Royaume-Uni, l'Australie, la Nouvelle-Zélande, les États-Unis et le Canada qui représentaient plus de $60 \%$ du volume total d'exportations de produits et services audiovisuels en 2000 (Thussu, 2008 ; Takhar, Maklran et Stevens, 2012). D'un autre côté, les films sont vendus dans des pays sensibles à l'esthétisme et au contenu des films indiens, tels qu'en Malaisie, Indonésie, Bahreïn, Thaïlande et aux Émirats arabes unis ${ }^{10}$ ou dans des pays, tels que la Chine et la Corée du Sud dont le marché cinématographique se trouve actuellement en pleine expansion. Toutefois, en 2014 les recettes en provenance du marché extérieur représentent que $7 \%$ des recettes totales du cinéma indien (OEA, $2015: 55)$.

17 À cela s'ajoute que les films d'auteur tiennent une place infime dans le paysage cinématographique indien et mondial. Durant la période entre 1955 et 2014, les films indiens n'ont réussi à remporter que quatre prix parmi les récompenses majeures des cinq grands festivals internationaux (Cannes, Venise, Berlin, San Sebastian, Locarno) ${ }^{11}$ : en 1957 Lion d'or en Venise ; en 1973 Ours d'Or en Berlin ; en 1981 Léopard d'or à Locarno 
et Ours d'argent en Berlin. À titre comparatif, sans aucun prix majeur jusqu'à la fin des années 1980, les films chinois en ont remporté 19 entre 1988 et 2014 dans les mêmes festivals (Vlassis, 2015a).

De façon générale, tenant compte de la production cinématographique de l'Inde, la place de son cinéma dans le marché cinématographique mondial reste marginale. En 2012, alors que la valeur des exportations des services audiovisuels de l'Inde atteint 303 millions USD contre 48 millions USD en 1998, les exportations de l'Union européenne (UE) s'élèvent à 6,2 milliards USD (OMC, 2014). En l'occurrence, certaines initiatives récentes tendent à stimuler le potentiel du cinéma indien à l'étranger: d'abord, l'accord de coopération entre des représentants de l'industrie cinématographique indienne et Hollywood, signé en 2010 en vue de créer un Los Angeles-India Film Council pour le renforcement de la production des films indiens aux États-Unis ; ensuite, en 2008 l'investissement de 550 millions USD de la société indienne Reliance - active dans le secteur des télécommunications - pour acquérir la moitié du capital de la société de production et de distribution des États-Unis DreamWorks (Augros, 2012 : 152-154) ; enfin, depuis les dix dernières années, la signature des traités de coproduction audiovisuelle du gouvernement indien avec un grand nombre de pays développés (Royaume-Uni, Canada, France, Allemagne, Italie, Espagne, Nouvelle-Zélande, Pologne) et des puissances dites « émergentes » (Brésil, Chine, Corée du Sud).

\section{L'Inde au sein du régime international de l'audiovisuel : entre stratégie modérée et alliances sporadiques}

Depuis le début des années 1990 et dans le contexte de l'expansion des échanges mondiaux des services et biens audiovisuels, des évolutions technologiques touchant « les industries imaginaires » (Flichy, 1991), ainsi que de la libéralisation de la radiodiffusion télévisuelle, un grand nombre d'acteurs ont débattu de la nature de la régulation des biens et services audiovisuels à l'échelle internationale (Vlassis et Richieri Hanania, 2014). À cet égard, le régime international de l'audiovisuel se fonde notamment sur deux instruments majeurs ${ }^{12}$ : d'un côté, l'Accord général sur le commerce des services (AGCS) de l'Organisation mondiale du commerce (OMC) et d'un autre côté, la Convention sur la protection et la promotion de la diversité des expressions culturelles (CDEC) adoptée par l'UNESCO en 2005 et ratifiée en mai 2016 par 143 États et l'UE (Vlassis, 2015b). La question qui se pose alors est de saisir le rôle et l'impact de l'Inde dans la construction et la mise en œuvre de ces instruments internationaux. Philippe Le Prestre (2005: 99) identifie quatre rôles distincts que sont censés jouer les États dans des négociations internationales : a) un rôle dirigeant, ou de chef de file (l'État cherche à faire avancer la coopération dans un sens qu'il préfère) ; b) un rôle d'appui ou de suiviste (l'État soutient les initiatives d'États chefs de file); c) un rôle de balancier (l'État exige d'importantes concessions à ses intérêts comme prix de son soutien); d) un rôle de frein, voire de blocage (l'État freine ou s'oppose à un accord soit par intransigeance, soit par refus de le mettre en œuvre). À cet égard, lors des négociations et de la mise en œuvre de l'AGCS et de la CDEC, les stratégies de l'Inde se caractérisent par un appui aux initiatives de chefs de file ou par un rôle de balancier. Pour autant, vu un paysage domestique autarcique, l'influence politique de la diplomatie indienne au sein du régime international de l'audiovisuel reste limitée. 


\subsection{L'Inde, l'intégration économique internationale et l'audiovisuel} de la libéralisation de l'audiovisuel. Restant réticente face à la position de l'exception culturelle défendue par la France, le Canada et plusieurs d'autres pays, elle est, parmi les dix-huit États ${ }^{13}$, qui a favorisé l'inclusion de l'audiovisuel dans l'agenda de l'AGCS lors de la dernière phase des négociations en 1993. D'ailleurs, l'Inde a cherché à exercer une pression en faveur d'une libéralisation du secteur audiovisuel auprès de pays, tels que l'Égypte ou l'Indonésie, où le public domestique reste attaché au langage particulier des films indiens (Mukherjee, 2005 : 248). Parallèlement, durant la période 2001-2005, l'Inde faisait partie du groupe informel de l'OMC 'Les Amis de l'Audiovisuel' (The Friends of Audiovisual) mis en place par les États-Unis et réunissant l'Argentine, le Brésil, l'Égypte, les États-Unis, Hong Kong, le Japon, le Mexique, la Nouvelle-Zélande, le Royaume-Uni et Taiwan ${ }^{14}$. Il s'agissait de viser à faire avancer les négociations sur les services audiovisuels ${ }^{15}$ au sein de l'AGCS. «À l'OMC, les États-Unis sont à la tête des initiatives. Tous les autres pays qui veulent libéraliser le secteur audiovisuel suivent parce qu'ils savent que les États-Unis consacrent des ressources et ils ont un intérêt vital. Les résultats d'une initiative sont pour tout le monde $»^{16}$.

Toutefois, les engagements pris par l'Inde au sein de l'OMC sont restreints; ils ne concernent que le secteur de la distribution de films et de cassettes vidéo en mode 3 (présence commerciale-établissement de filiales ou de succursales par une entreprise étrangère en vue de la fourniture de services dans un autre pays) et mode 4 (présence des personnes physiques pour fournir des services dans un autre pays membre de l'OMC). En effet, en matière de présence commerciale, l'Inde a ouvert son marché aux entreprises étrangères sous deux conditions : que ces entreprises agissent à travers des bureaux de représentation autorisés à fonctionner en tant que filiales d'entreprises constituées en dehors du territoire indien; et que l'importation de titres soit restreinte à 100 par an, même si depuis les années 1990 le nombre de films importés est en réalité beaucoup plus élevé. D'ailleurs, l'Inde - comme plusieurs d'autres pays - s'est distanciée des initiatives plus récentes du groupe des amis de l'audiovisuel. Elle n'a pas signé en 2005 la dernière déclaration du groupe au sujet d'une demande plurilatérale relative à l'avancement des négociations sur les services audiovisuels, distribuée auprès des membres de l'OMC par les délégations de Hong Kong, Japon, Mexique, Taiwan et États-Unis. À cela s'ajoute que la question du traitement des biens et services culturels dans les accords commerciaux multilatéraux et bilatéraux n'est pas dans les priorités de la diplomatie indienne. Il est révélateur que les accords de libre-échange (ALE) conclus par l'Inde avec le Chili (2006), la Corée du Sud (2009) et le Japon (2011) n'attribuent aucun statut particulier aux biens et services culturels.

\subsection{L'Inde vis-à-vis de l'enjeu de la diversité des expressions culturelles}

Lors des négociations en vue de l'adoption de la CDEC, l'Inde a défendu deux positions majeures : défense de la souveraineté étatique en matière d'industries culturelles et mise en place d'un instrument juridique international peu contraignant conservant une marge

Revue Interventions économiques, 55 | 2016 
de manœuvre considérable pour les États parties dans sa mise en application (Vlassis, 2011).

D'une part, à la suite des chefs de file de la CDEC comme la France et le Canada, la délégation indienne s'est penchée sur l'importance des politiques culturelles et du rôle des États dans la protection et la promotion de la diversité des expressions culturelles, ainsi que sur l'aspect non commercial des biens et services culturels (UNESCO, 2004c : 30). D'autre part, elle s'est opposée, avec des pays tels que les États-Unis, le Japon et la Nouvelle-Zélande, à l'adoption d'une Convention qui ferait contrepoids au régime de l'OMC. Ainsi, l'Inde était-elle favorable à la variante B de l'article 19 de l'avant-projet de la Convention selon laquelle « rien, dans la présente Convention, ne modifie les droits et obligations des États parties au titre d'autres instruments internationaux existants ». D'ailleurs, une question est devenue prioritaire dans l'agenda indien: s'opposer à des parties tierces à l'accord de recevoir autorité pour interpréter les normes de la CDEC et de suivre leur mise en œuvre. À cet égard, l'Inde est un des quatre pays - avec les États-Unis, le Japon, et la Turquie - qui s'est ouvertement opposée à l'inclusion d'un mécanisme de règlement des différends dans la $\mathrm{CDEC}$ puisque ce type de mécanisme est selon elle "inutile, inopportun et anachronique » (UNESCO, 2004a: 109) dans le cadre d'une convention destinée à sauvegarder la diversité culturelle. À la suite des fortes objections de ces quatre pays, l'article 25 du texte final de la CDEC inclut le paragraphe 4 selon lequel " chaque Partie peut, au moment de la ratification, de l'acceptation, de l'approbation ou de l'adhésion, déclarer qu'elle ne reconnaît pas la procédure de conciliation prévue cidessus » (UNESCO, 2005). Dans le même esprit, le gouvernement indien a explicitement suggéré la suppression de l'article sur l'établissement d'un Observatoire de la diversité culturelle - position recommandée par le groupe d'experts indépendants -, estimant que ce dispositif est censé « diluer le rôle de l'État et ses fonctions » et que, par conséquent, « il ne cadre pas avec l'esprit de la Convention » (UNESCO, 2004c : 31).

En ce qui concerne l'article 6 de l'avant-projet qui prévoit les droits et les obligations des États parties au niveau national, l'Inde a recommandé la suppression du terme "obligations", ainsi que de l'expression "adopter les mesures règlementaires et financières", puisque ce genre de détail "doit être laissé à la discrétion des États Parties» (UNESCO, 2004b: 52). En outre, l'Inde s'est distanciée du débat autour du renforcement de la coopération culturelle internationale et de la mise en place d'un Fonds international pour la diversité culturelle (FIDC). L'Inde s'est en réalité montrée réticente à l'idée de se lier les mains de manière trop rigide sur des questions sensibles comme le règlement des différends, les liens de la $\mathrm{CDEC}$ avec les accords commerciaux, les mécanismes destinés à la coopération culturelle internationale ou les politiques appropriées pour la protection et la promotion de la diversité des expressions culturelles.

Enfin, tandis que l'Inde est partie à la CDEC depuis 2006, elle est peu impliquée dans le processus de sa mise en œuvre. Dans le cadre de l'article 9 de la CDEC, les parties fournissent tous les quatre ans des rapports périodiques sur les mesures prises en vue de protéger et promouvoir la diversité des expressions culturelles sur leur territoire et au niveau international (Vlassis, 2011). La soumission du rapport quadriennal de l'Inde, prévue pour 2012, a eu lieu en septembre 2015 pour des raisons de manque d'expertise et de ressources humaines de la part des autorités indiennes en vue de collecter et synthétiser les informations nécessaires. À cela s'ajoute que le rapport soumis recense notamment des politiques et des mesures moins relatives aux industries culturelles que relatives à la conservation du patrimoine culturel qui n'est pas l'objet de la Convention de 
2005 (UNESCO, $2015: 14$ ). Un autre signe de la faible implication de l'Inde est la mise en œuvre du FIDC (Vlassis, 2014) destiné à soutenir les industries culturelles des pays moins avancés : la contribution de l'Inde atteint en mai 201645000 USD, alors que celle d'autres pays dits «émergents " reste beaucoup plus régulière et importante : celle de la Chine s'élève à 310000 USD, celle du Mexique à 362000 USD, et celle du Brésil à 300000 USD.

\section{L'Inde et la compétition mondiale : le cinéma populaire indien au moment du « cinéma-monde »}

Inspiré par l'historien français Fernand Braudel et son concept d'économie-monde, Charles-Albert Michalet (1987) a montré que depuis les années 1980 Hollywood a développé la stratégie du "cinéma-monde » fondée sur trois piliers : d'abord, un filmmonde, à la fois film évènement et film universel, destiné à tous les publics et à tous les pays ; ensuite, les majors hollywoodiennes adoptent une approche mondiale du marché nécessaire à l'amortissement de ces nouvelles superproductions ; enfin, les majors sont obligées de renoncer à leur autonomie afin d'établir une union étroite avec des groupes conglomérats sur la base d'une concertation horizontale des sociétés centrée autour des activités des loisirs. Comme l'a expliqué C.-A. Michalet, le cinéma-monde s'inscrit comme une réponse des majors hollywoodiennes face aux défis de l'ère postfordiste et au référentiel de la dérégulation des marchés. En ce sens, «le cinéma-monde reflète les forces économiques qui font émerger un capitalisme mondial, c'est-à-dire, un système économique qui ne peut fonctionner que dans la dimension planétaire » (Michalet, 1987 : 112).

Les exportations des services audiovisuels américains s'élèvent alors à 13,5 milliards USD en 2010, beaucoup plus que celles de l'UE - qui sont en grande partie intra-européennes -, du Canada ou des pays dits « émergents » (Table II). Par ailleurs, la balance commerciale de l'industrie audiovisuelle américaine est depuis longtemps positive et en hausse constante, bien que les États-Unis souffrent d'un déficit commercial chronique. En 2012 ce surplus a atteint 13,5 milliards USD (Table III), plus que le surplus des services de télécommunications, du management/consulting, des secteurs légaux, médicaux ou informatiques (United States International Trade Commission, 2014). Plus remarquable encore, pendant que plusieurs secteurs industriels des États-Unis, tels que l'aéronautique, l'automobile, l'agroalimentaire, la raffinerie ou l'informatique-électronique, doivent faire face à une concurrence de la part de leurs homologues internationaux - surtout asiatiques et européens -, les majors hollywoodiennes n'ont véritablement aucun concurrent à l'échelle mondiale. Bénéficiant de développements technologiques fondamentaux, le film hollywoodien demeure également un produit clé pour les supports électroniques (DVD, télévision, Internet).

De ce fait, tandis que les spécificités culturelles, un langage filmique singulier ou les mesures financières et règlementaires en matière de cinéma réussissent à sauvegarder et promouvoir une industrie cinématographique nationale dans un grand nombre de pays comme en France, en Corée du Sud, en Chine, au Japon ou en Inde (OEA, 1998-2013), ils ne parviennent pas à remettre en question la prééminence de Hollywood en termes de distribution et d'exportation à l'échelle mondiale (Trumbour, 2008 ; Crane, 2013 ; Vlassis, 2015a). 
29 Il est révélateur que durant la période 2010-2015, dix-huit grosses productions hollywoodiennes ont enregistré plus de $75 \%$ de leurs recettes globales sur des marchés hors des États-Unis et du Canada (Table IV). En outre, d'après la liste des plus gros succès du box-office mondial incluant 400 films (Mojo, 2014), nous ne retrouvons que sept productions non-hollywoodiennes, dont 5 films anglophones, mais aucune production indienne: Lucy (2014), Intouchables (2011), The King's Speech (2010), Slumdog Millionaire (2008), Taken 2 (2012), Resident Evil : Afterlife (2010), Spirited Away (2001). Parmi ces films, six sont des productions britanniques, allemandes et françaises ${ }^{17}$, et une seule japonaise (le film d'animation Spirited Away). Cela témoigne du savoir-faire, de l'avance technologique et de la capacité de financement des majors d'Hollywood pour commercialiser des films à l'échelle mondiale. Malgré leurs rivalités, les majors sont reliées par un langage filmique commun et par des interdépendances stratégiques fortes et ils bénéficient des économies d'échelle et de la popularité de la langue anglaise (Augros et Kitsopanidou, 2009 ; Vlassis, 2015a).

À cela s'ajoute que la part du film étranger reste extrêmement infime dans le marché cinématographique des États-Unis, allant de $4 \%$ à $9 \%$. Comme le révèle la table $\mathrm{V}$, tout au long de la période 1994-2015, seulement 20 productions non étatsuniennes, dont 17 européennes, parviennent à enregistrer des recettes non négligeables, à savoir plus de 40 millions USD, dans le marché cinématographique des États-Unis. D'ailleurs, deux productions indiennes - P.K. (2014) et Monsoon Wedding (2001) - réussissent à enregistrer plus de 10 millions USD de recettes.

31 Enfin, les tables comparatives VI et VII sur les recettes totales des dix productions indiennes et hollywoodiennes illustrent nettement quatre points: (i) la mainmise incontestable d'Hollywood dans le marché mondial des biens cinématographiques, et surtout dans certains marchés majeurs comme celui de la Chine ; (ii) l'écart écrasant entre le cinéma hollywoodien et les films indiens dans les marchés internationaux ; (iii) la faiblesse structurelle du cinéma indien de s'exporter ; (iv) la capacité restreinte des majors hollywoodiennes de pénétrer le marché cinématographique indien.

Le "cinéma-monde " contribue donc à consolider l'asymétrie des flux dans l'économie audiovisuelle mondiale au bénéfice d'Hollywood et à asseoir la domination du cinéma hollywoodien dans deux sens : d'une part, grâce à un marché domestique fondé sur des investissements colossaux, des films-évènements fort médiatiques, la puissance financière des majors hollywoodiennes et leur force sans égal dans la distribution, le " cinéma-monde » sert à barrer l'accès au marché des États-Unis aux producteurs et distributeurs étrangers qui ne bénéficient pas de ressources financières et humaines substantielles pour mettre en œuvre des stratégies propres à Hollywood ; d'autre part, selon la même logique, il attribue l'avantage aux films hollywoodiens dans les échanges mondiaux, contribuant à une coordination des marchés nationaux et à une convergence progressive des préférences cinématographiques des spectateurs (Scott, 2004 ; Laroche et Bohas, 2005).

\section{Conclusion}

La sphère internationale et la sphère nationale sont complémentaires et elles nous révèlent la place de l'Inde dans la distribution mondiale des ressources intangibles de puissance. Il convient alors de tirer quatre conclusions. En premier lieu, l'industrie 
cinématographique indienne est peu organisée et fortement fragmentée et, d'un autre, l'intervention de l'État dans le secteur est discrète et minime. L'Inde se caractérise par un paysage cinématographique peu structuré, une production cinématographique en série qui est dispersée en plusieurs pôles, des pouvoirs publics peu interventionnistes. Le cinéma indien est en grande partie doté d'un langage filmique dominant et conventionnel qui est particulier et adapté aux goûts culturels du public indien. D'un côté, le langage filmique du cinéma indien et une production en série prolifique tendent à dominer largement le paysage cinématographique du pays. Par conséquent, le marché cinématographique indien est impénétrable face aux tentatives des majors d'Hollywood et d'autres distributeurs étrangers. D'un autre côté, vu des particularités domestiques du cinéma indien, les films indiens sont très peu exportables et n'arrivent pas à conquérir les marchés audiovisuels majeurs, au moins en termes de recettes (qu'il s'agisse de l'Amérique du Nord, de l'Europe, de l'Australie, du Brésil, du Mexique, de la Russie, du Japon, de la Chine, de la Corée du Sud).

En second lieu, absorbée par un système cinématographique autarcique, l'Inde pèse très peu dans la circulation des biens et services audiovisuels à l'échelle mondiale. Se conformant à l'architecture institutionnelle du régime international de l'audiovisuel, elle se contente d'un rôle de suiviste ou de balancier dans la confrontation normative du régime de libéralisation des échanges audiovisuels et les tentatives de promotion ou de protection de la diversité des expressions culturelles. D'ailleurs, dans la mesure où les coalitions du régime n'obéissent pas à une logique Nord/Sud ou pays développés/ puissances émergentes, l'Inde entretient des alliances sporadiques sur des questions spécifiques comme le règlement des différends, le degré de la libéralisation de l'audiovisuel ou la coopération culturelle internationale.

Autre variable importante, considérée comme un objet référentiel, Hollywood dispose encore d'un savoir-faire inégalé. Ses stratégies de distribution mondiales et sa capacité de financement et d'innovation unique sont sans rival. "Les productions hollywoodiennes qui représentent les seuls symboles commercialisés à l'échelle planétaire, façonnent les imaginaires des spectateurs » (Laroche, $2013: 644$ ).

En d'autres termes, nos conclusions vont à l'encontre de l'hypothèse selon laquelle l'Inde veut et peut jouer un rôle dans la bataille mondiale de l'attraction. Contrairement à ce qu'on pourrait croire, le cinéma n'offre pas à l'Inde un instrument de soft power et celle-ci ne cherche pas à le mettre au niveau de ses concurrents existants et potentiels, manifestant ainsi une exception indienne fondée sur ses particularités institutionnelles et culturelles. Par conséquent, la machine diplomatique de l'Inde ne vise pas à déployer des ressources substantielles afin de construire un certain statut de puissance culturelle pour le pays. Elle aborde les questions multilatérales sous le prisme des spécificités de la sphère domestique et sa présence au sein des arènes multilatérales touchant des enjeux sur les industries culturelles reste alors restreinte. À cet égard, l'Inde est encore loin de s'imposer en tant puissance culturelle régionale, voire mondiale et de modifier les rapports de force au sein de l'économie mondiale des industries imaginaires. 
Tableau 1. Part du cinéma national (en \%)

\begin{tabular}{llll}
\hline Pays/Date & $\mathbf{2 0 0 9}$ & $\mathbf{2 0 1 2}$ & $\mathbf{2 0 1 4}$ \\
\hline Inde & 92,0 & 91,5 & 91,5 \\
Chine & 56,6 & 48,5 & 54,5 \\
Japon & 56,9 & 65,7 & 58,3 \\
Corée du Sud & 48,8 & 58,8 & 50,1 \\
France & 36,8 & 40,2 & 44,4 \\
\hline
\end{tabular}

Source : Observatoire européen de l'audiovisuel

Tableau 2. Exportateurs et importateurs majeurs des services audiovisuels, 2010 et 2011 (millions USD et pourcentage)

\begin{tabular}{|c|c|c|c|c|c|c|c|}
\hline & \multicolumn{2}{|c|}{ Valeur } & \multirow{2}{*}{$\begin{array}{l}\text { Part dans } \\
\text { les } 10 \\
\text { économies } \\
2010 \\
20\end{array}$} & \multicolumn{4}{|c|}{ Changement annuel } \\
\hline & 2010 & 2011 & & $\begin{array}{l}2005- \\
10\end{array}$ & 2009 & 2010 & 2011 \\
\hline \multicolumn{8}{|l|}{ Exportateurs } \\
\hline États-Unis & 13529 & $\ldots$ & 48,1 & 6 & 4 & -2 & ... \\
\hline Union européenne (27) & 11203 & $\ldots$ & 39,8 & 3 & 2 & 11 & ... \\
\hline Dont extra-UE (27) exportations & 4802 & $\ldots$ & 17,1 & 2 & 9 & 8 & $\ldots$ \\
\hline Canada & 1954 & 2233 & 6,9 & 1 & -5 & 5 & 14 \\
\hline Russie & 360 & 310 & 1,3 & 23 & -1 & 39 & -14 \\
\hline Argentine & 343 & 298 & 1,2 & 12 & -41 & 25 & -13 \\
\hline Norvège & 204 & ... & 0,7 & 1 & -9 & -20 & ... \\
\hline Corée du Sud & 189 & 271 & 0,7 & 8 & -5 & -5 & 44 \\
\hline Australie & 128 & 209 & 0,5 & 0 & -45 & 19 & 63 \\
\hline Chine & 123 & 147 & 0,4 & -2 & -77 & 26 & 19 \\
\hline \multirow[t]{2}{*}{ Hong Kong, Chine } & 112 & $\ldots$ & 0,4 & -14 & -50 & -2 & $\ldots$ \\
\hline & 28145 & $\ldots$ & 100,0 & - & - & - & - \\
\hline \multicolumn{8}{|l|}{ Importateurs } \\
\hline Union européenne (27) & 13356 & $\ldots$ & 60,5 & 0 & -8 & 3 & $\cdots$ \\
\hline Dont Extra-UE (27) importations & 5699 & $\ldots$ & 25,8 & -3 & -15 & 3 & ... \\
\hline Canada & 2393 & 2445 & 10,8 & 7 & -3 & 30 & 2 \\
\hline États-Unis & 1666 & $\ldots$ & 7,5 & 12 & 8 & -13 & $\ldots$ \\
\hline Australie & 1031 & 1332 & 4,7 & 12 & -18 & 15 & 29 \\
\hline Brésil & 957 & 1043 & 4,3 & 25 & 23 & 32 & 9 \\
\hline Russie & 851 & 875 & 3,9 & 18 & 6 & 18 & 3 \\
\hline Japon & 732 & $\ldots$ & 3,3 & -4 & -12 & -11 & ... \\
\hline Chine & 371 & 371 & 1,7 & 19 & 9 & 33 & 0 \\
\hline Corée du Sud & 360 & 329 & 1,6 & 18 & -7 & 12 & -9 \\
\hline \multirow[t]{2}{*}{ Argentine } & 356 & 428 & 1,6 & 17 & 14 & 27 & 20 \\
\hline & 22070 & $\ldots$ & 100,0 & - & - & - & - \\
\hline
\end{tabular}

Source : OMC

Tableau 3. Services audiovisuels : Exportations et importations des États-Unis, 2008-2012 (en milliards USD)

\begin{tabular}{llllll}
\hline & $\mathbf{2 0 0 8}$ & $\mathbf{2 0 0 9}$ & $\mathbf{2 0 1 0}$ & $\mathbf{2 0 1 1}$ & $\mathbf{2 0 1 2}$ \\
\hline Exportations & 13,2 & 13,7 & 13,6 & 14,5 & 16,2 \\
Importations & 1,7 & 1,9 & 1.6 & 2,0 & 2,6 \\
$\begin{array}{l}\text { Balance } \\
\text { commerciale }\end{array}$ & 11,4 & 11,8 & 12,0 & 12,5 & 13,5 \\
\hline
\end{tabular}

Source : US Trade Commission 
Tableau 4. Films hollywoodiens et recettes internationales, 2010-2015

\begin{tabular}{ll}
\hline Film (2010-2015) & $\begin{array}{l}\text { \% de ses recettes globales dans les marches hors des } \\
\text { Etats-Unis et du Canada }\end{array}$ \\
\hline Gulliver's Travel (2010) & 82,0 \\
Ice Age: Continental Drift (2012) & 81,6 \\
The Smurfs 2 (2013) & 79,6 \\
Life of Pi (2012) & 79,5 \\
The Adventures of Tintin (2011) & 79,3 \\
Battleship (2012) & 78,4 \\
A Good Day to Die Hard (2013) & 77,9 \\
Transformers: Age of Extinction (2014) & 77,4 \\
Spectre (2015) & 77,3 \\
Pirates of the Caribbean: On Stranger & 76,9 \\
Tides (2011) & 76,7 \\
Furious 7 (2015) & 75,9 \\
RoboCop (2014) & 75,7 \\
The Tourist (2010) & 75,7 \\
American Reunion (2012) & 75,4 \\
Hansel and Gretel: Witch Hunters & 75,2 \\
(2013) & 75,2 \\
Pacific Rim (2013) & 75,2 \\
After Earth (2013) & \\
Kung Fu Panda 2 (2011) &
\end{tabular}

Source: Mojo

Tableau 5. Recettes des productions cinématographiques non-américaines, 1994-2015 (en millions USD)

\begin{tabular}{llll}
\hline Film & $\begin{array}{l}\text { Recettes/ } \\
\text { Etats-Unis- } \\
\text { Canada }\end{array}$ & $\begin{array}{l}\text { Recettes/ } \\
\text { International }\end{array}$ & Total \\
\hline Taken (France-2008) & 145,0 & 81,8 & 226,8 \\
Slumdog Millionaire (Royaume-Uni-2008) & 141,3 & 236,5 & 377,9 \\
Taken II (France-2012) & 139,8 & 236,2 & 376,1 \\
The King's speech (Royaume-Uni-2010) & 138,7 & 275,4 & 414,2 \\
Crouching Tiger, Hidden Dragon (Taiwan/Hong Kong- & 128,0 & 85,4 & 213,5 \\
2000) & 126,6 & 332,2 & 458,8 \\
Lucy (France-2014) & 89,2 & 237,2 & 326,4 \\
Taken 3 (France-2015) & 77,4 & 49,9 & 127,3 \\
La marche de I'empereur (France-2005) & 63,8 & 200,1 & 263,9 \\
The fifth element (France-1997) & 60,1 & 236,0 & 296,2 \\
Resident Evil : Afterlife (France-Allemagne-2010) & 57,5 & 171,6 & 229,1 \\
La vita è bella (Italie-1997) & 56,4 & 66,9 & 123,3 \\
The Queen (France-Royaume-Uni-2006) & 53,7 & 123,6 & 177,3 \\
Hero (Chine-2002) & 52,7 & 193,0 & 245,7 \\
Four weddings and a funeral (Royaume-Uni-1994) & 51,2 & 78,1 & 129,3 \\
Resident evil : Extinction (France-Allemagne-2004) & 45,9 & 211,9 & 257,9 \\
The Full Monty (Royaume-Uni-1997) & 45,3 & 205,8 & 251,2 \\
Bean (Royaume-Uni-1997) & 45,0 & 37,6 & 82,7 \\
28 days later (Royaume-Uni-2003) & 44,6 & 88,7 & 133,4 \\
The Artist (France-2012) & 44,4 & 54,6 & 99,0 \\
No se aceptan devoluciones (Mexique-2013) & 43,0 & 42,0 & 85,1 \\
\hline The Transporter 2 (France-2005) & & & \\
\hline
\end{tabular}

Source : Mojo/IMDB 
Tableau 6. Recettes des productions indiennes (en millions USD)

\begin{tabular}{|c|c|c|c|c|c|c|c|}
\hline Film & $\begin{array}{l}\text { Recettes } \\
\text { totales }\end{array}$ & $\begin{array}{l}\text { Dont } \\
\text { États- } \\
\text { Unis/Canada }\end{array}$ & $\begin{array}{l}\text { Royaume- } \\
\text { Uni }\end{array}$ & Australie & Chine & $\begin{array}{l}\text { Corée } \\
\text { du Sud }\end{array}$ & $\begin{array}{l}\text { Émirats } \\
\text { arabes } \\
\text { unis }\end{array}$ \\
\hline P.K. (2014) & 100,3 & 10,6 & 3,1 & 1,7 & 19,3 & - & - \\
\hline $\begin{array}{l}\text { Dhoom } 3 \\
(2013)\end{array}$ & 88,0 & 8,0 & 4,4 & 1,5 & 2,9 & - & - \\
\hline $\begin{array}{l}\text { Chennai } \\
\text { Express } \\
\text { (2013) }\end{array}$ & 68,0 & 5,3 & 3,3 & 0.6 & - & - & - \\
\hline Kick (2014) & 64,0 & 2,4 & 2,1 & 0,4 & - & - & - \\
\hline $\begin{array}{l}3 \text { idiots } \\
\text { (2009) }\end{array}$ & 61,0 & 4,2 & 2,5 & 0,9 & - & 3,0 & - \\
\hline $\begin{array}{l}\text { Krrish } 3 \\
\text { (2013) }\end{array}$ & 61,0 & 2,1 & 1,3 & 0,4 & - & - & - \\
\hline $\begin{array}{l}\text { Prem Ratan } \\
\text { Dhan Payo } \\
\text { (2015) }\end{array}$ & 52,5 & 4,3 & 2,2 & 0,6 & & & 2,2 \\
\hline $\begin{array}{l}\text { My name is } \\
\text { Khan (2010) }\end{array}$ & 42,3 & 4,0 & 3,5 & 0,7 & - & 2,6 & 2,1 \\
\hline $\begin{array}{l}\text { Bang Bang! } \\
(2014)\end{array}$ & 42,0 & 2,5 & 0,9 & 0,2 & - & - & 3,3 \\
\hline $\begin{array}{l}\text { Ghajini } \\
\text { (2008) }\end{array}$ & 38,3 & 2.4 & 1,0 & 0,3 & - & - & - \\
\hline
\end{tabular}

Source : Mojo/IMDB

Tableau 7. Recettes des productions hollywoodiennes (en millions USD)

\begin{tabular}{|c|c|c|c|c|c|c|c|}
\hline Film & $\begin{array}{l}\text { Recettes } \\
\text { totales }\end{array}$ & $\begin{array}{l}\text { Dont } \\
\text { Inde }\end{array}$ & $\begin{array}{l}\text { Royaume- } \\
\text { Uni }\end{array}$ & Australie & Chine & $\begin{array}{l}\text { Corée } \\
\text { du Sud }\end{array}$ & $\begin{array}{l}\text { Émirats } \\
\text { arabes unis }\end{array}$ \\
\hline Avatar (2009) & 2787,9 & 24,2 & 150,0 & 105,7 & 204,1 & 105,4 & 7,3 \\
\hline Titanic (1997) & 2186,7 & 12,7 & 114,0 & 38,8 & 43,9 & 17,2 & 2,9 \\
\hline Jurrasic World (2015) & 1670,4 & 22,2 & 99,6 & 38,9 & 228,7 & 42,8 & 7,8 \\
\hline $\begin{array}{l}\text { Marvel's Avengers } \\
\text { (2012) }\end{array}$ & 1519,5 & 12,6 & 80,5 & 54,3 & 86,3 & 50,6 & 6,6 \\
\hline Furious 7 (2015) & 1516,0 & 24,7 & 59,9 & 39,9 & 390,9 & 24,0 & 2,3 \\
\hline $\begin{array}{l}\text { Avengers : Age of } \\
\text { Ultron (2015) }\end{array}$ & 1405,4 & 16,6 & 76,6 & 30,9 & 240,1 & 78,2 & 3,1 \\
\hline $\begin{array}{l}\text { Harry Potter and the } \\
\text { Deathly Hallows } 2 \\
\text { (2011) }\end{array}$ & 1341,5 & 7,7 & 117,2 & 51,3 & 60,8 & 32,0 & 2,9 \\
\hline Frozen (2011) & 1274,2 & 0,6 & 64,7 & 31,0 & 48,2 & 76,6 & 3,1 \\
\hline Iron Man 3 (2013) & 1215,4 & 12,2 & 57,0 & 36,1 & 121,2 & 64,2 & 5,7 \\
\hline Minions (2015) & 1159,0 & 2,6 & 72,6 & 23,4 & 68,4 & 15,3 & 4,6 \\
\hline
\end{tabular}

Source : Mojo/IMDB 


\section{BIBLIOGRAPHIE}

Augros, Joël (2011). « Bollywood vs. Hollywood », CinémAction, n¹38, mars, pp. 104-110.

Augros, Joël (2012). « De Hollywood à Bollywood », Théorème, n¹6, pp. 143-159.

Augros, Joël et Kira Kitsopanidou (2009). L'économie du cinéma américain : histoire d'une industrie culturelle et de ses stratégies, Paris, Armand Colin, 286 pages.

Battistella, Dario (2013). « Puissance, vers une approche synthétique ? », dans Dario Battistella (sous la direction de), Relations internationales, Bilan et perspectives, Paris, Ellipses, pp. 237-260.

Bohas, Alexandre (2010). Disney, un capitalisme mondial du rêve, Paris, L'Harmattan, 248 pages.

Bohas, Alexandre (2006). « The Paradox of Anti-Americanism : Reflection on the Shallow Concept of Soft Power ", Global Society, vol. 20, n4, octobre, pp. 395-414.

Brewster, David Halstead (2012). India as an Asia Pacific Power, London, New York, Routledge.

Central Board of Film Certification (2011). Annual Report 2011, Ministry of Information \& Broadcasting, Government of India. Consulté sur Internet (http://cbfcindia.gov.in/html/ uniquepage.aspx?unique_page_id=30) le 06 juin 2015.

Chavagneux, Christian (2010). Économie politique internationale, Paris, La Découverte, $2^{\mathrm{e}}$ édition, 126 pages.

Chitrapu, Sunitha (2013). « The big stick behind 'soft power' ? The case of Indian films in international markets ", dans David Shafaer, Kavita Karan (sous la direction de), Bollywood and Globalization: Global Power of Popular Hindi Cinema, London, New York, Routledge, pp. 15-28.

Crane, Diana (2013). «Cultural globalization and the dominance of the American film industry: cultural policies, national film industries, and transnational film », International journal of cultural policy, septembre, (publié en ligne). Consulté sur Internet (http://www.tandfonline.com/doi/ full/10.1080/10286632.2013.832233\#.Ux9All7yuCc) le 13 mai 2015.

Dagnaud, Monique (2009). « Les industries de l'image. Le cinéma en Inde, aux États-Unis et en France », La vie des idées.fr (revue électronique). Consulté sur Internet (http://www.laviedesidees.fr/ Les-industries-de-l-image.html) le 19 juin 2015.

Dagnaud, Monique (2011). «Le cinéma, instrument du soft power des nations ", Revue française de Géoéconomie, n58, pp. 21-30.

Deprez, Camille (2010). Bollywood, cinéma et mondialisation, Villeneuve d'Ascq, Presses universitaires du Septentrion, 252 pages.

Deprez, Camille (2011). « La production cinématographique indienne : entre diversité régionale et hybridité », CinémAction, n¹38, mars, pp. 114-118.

Dupont, Olivier (2009). «Le défi indien : entre pluripolarité et pragmatisme réaliste », dans Sebastian Santander (sous la direction de), L'émergence des nouvelles puissances, vers un système multipolaire? Paris, Ellipses, pp. 129-170.

Farges, Joël (2000). « Le cinéma populaire indien : un parfum d'opium... », Critique internationale, n ${ }^{\circ} 7$, pp. 157-168. 
Farges, Joël (2006). « Le cinéma en Inde : Rasa cinematografica », dans Christophe Jaffrelot (sous la direction de), L'Inde contemporaine : de 1950 à nos jours, Paris, Fayard, CERI, pp. 689-716.

Flichy, Patrice (1991). Les industries imaginaires, pour une analyse économique des médias, Grenoble, Presses universitaires de Grenoble, $2^{e}$ édition, 277 pages.

Ganti, Tejaswini (2012). Producing Bollywood, Inside the Contemporary Hindi Film Industry, Durham, NC, Duke University Press, 424 pages.

Hymans, Jacques (2009). «India's Soft Power and Vulnerability », India Review, vol. 8, n³, pp. 234-265.

Jaffrelot, Christophe (2008). New Delhi et le monde: une puissance émergente entre realpolitic et soft power, Paris, Autrement, 156 pages.

Jaffrelot, Christophe (2006). Introduction, dans Christophe Jaffrelot (sous la direction de), L'Inde contemporaine : de 1950 à nos jours, Paris, Fayard, CERI, pp. 11-21.

Jain, Bakhtavar Mal (2008). Global power : India's foreign policy 1947-2006, Lanham, Lexington Books, 326 pages.

Kapur, Ashok (2006). India from regional to world power, London, Routledge, 253 pages.

Katzenstein, Peter J. (2005). A world of regions : Asia and Europe in the American Imperium, Ithaca, Cornell University Press, 320 pages.

Kugiel, Patryk (2012). «India’s Soft Power in South Asia », International Studies, vol. 49, n³-4, pp. 351-376.

Laroche, Josepha (2013). « L'économie politique internationale », dans Thierry Balzacq et Fréderic Ramel (sous la direction de), Traité de relations internationales, Paris, Presses de Sciences Po, pp. 631-659.

Laroche, Josépha et Alexandre Bohas (2005). Canal+ et les majors américaines, une vision désenchantée du cinéma-monde, Paris, Pepper éditions, 271 pages.

Le Prestre, Philippe (2005). Protection de l'environnement et relations internationales : les défis de l'écopolitique mondiale, Paris, Armand Colin, 477 pages.

Matusitz, Jonathan (2012). « Globalisation of Popular Culture : From Hollywood to Bollywood », South Asia Research, vol. 32, n², July, pp. 123-138.

Michalet, Charles-Albert (1987). Le drôle de drame du cinéma mondial : une industrie culturelle menacée, Paris, La Découverte, 214 pages.

Mingant, Nolwenn (2010). Hollywood à la conquête du monde. Marchés, stratégies, influences, Paris, CNRS éditions, 316 pages.

MOJO (2014). All time box office, Worldwide Grosses, Consulté sur Internet (http:// boxofficemojo.com/alltime/world/?pagenum=1\&p=.htm) le 10 juin 2015.

Morin, Jean-Frédéric (2013). La politique étrangère. Théories, méthodes et références, Paris, Armand Colin, 315 pages.

Mukharjee, Arpita (2005). " Audio-visual policies and international trade : the case of India ", dans Paolo Guerrieri, P. Lelio Iapadre, Georg Koopmann (sous la direction de), Cultural diversity and international economic integration : the global governance of the audio-visual sector, Northampton, Edward Elgar, pp. 218-258.

Mukharjee, Arpita (2002). « India's Trade Potential in Audiovisual Services and the GATS », Indian Council for Research on International Economic Relations, Working Paper $n^{\circ} 81$. Consulté sur Internet ( 
http://www.icrier.org/pdf/avpaper.pdf) le 05 juin 2015. Nayar, Baldev Raj (2003). India in the world order : searching for major-power status, Cambridge, Cambridge University Press, 291 pages.

NFDC (National Film Development Corporation), 2014. Annual Report 2012-2013. Consulté sur Internet (http://www.nfdcindia.com/pdf/AnnualReport2013.pdf) le 09 février 2016.

Nye, Joseph S. (1990). Bound to lead : the changing nature of American power, New York, Basic Books, 336 pages.

Nye, Joseph S. (2004). Soft power, the means to success in world politics, New York, Public Affairs, 191 pages.

OEA (Observatoire européen de l'audiovisuel), (2015). FOCUS, Tendances du marché mondial du film, Strasbourg, OEA.

OEA (Observatoire européen de l'audiovisuel), (1998-2013). FOCUS, Tendances du marché mondial du film, Strasbourg, OEA.

OMC (Organisation mondiale du commerce), (2014). International Trade Statistics. Consulté sur Internet (http://www.wto.org/english/res_e/statis_e/its2014_e/its14_toc_e.htm) le 03 juin 2015.

Pahlavi, Pierre (2013). « La diplomatie publique », dans Thierry Balzacq, Fréderic Ramel, (sous la direction de), Traité de relations internationales, Paris, Presses de Sciences Po, pp. 553-605.

Panagariya, Arvind (2008). India, the emerging giant, Oxford, Oxford University Press, 546 pages.

Paquin, Stéphane (2013). Théories de l'économie politique internationale : cultures scientifiques et hégémonie américaine, Paris, Presses de Sciences Po, 359 pages.

Pendakur, Manjunath (1996). «India's National Film Policy : Shifting Currents in the 1990s », dans Albert Moran (sous la direction de), Film Policy : International, National and Regional Perspectives, Londres, Routledge, pp. 145-168.

Santander, Sebastian (2013). «Les puissances émergentes, portée et limites d'un phénomène international », dans Dario Battistella (sous la direction de), Relations internationales, Bilan et perspectives, Paris, Ellipses, pp. 523-544.

Scott, Allen J. (2004). « Hollywood and the World : the Geography of Motion-Picture Distribution ", Review of International Political Economy, vol. 11, n¹, pp. 33-61.

Shafaer, David J. et Kavita Karan (sous la direction de) (2013). Bollywood and Globalization : Global Power of Popular Hindi Cinema, London, New York, Routledge, 208 pages.

Strange, Susan (1994). States and Markets : An Introduction to International Political Economy, London, Frances Pinter, $2^{\mathrm{e}}$ edition, 280 pages.

Takhar, Amandeep Pauline Maclaran et Lorna Stevens (2012). «Bollywood Cinema's Global Reach : Consuming the 'Diasporic Consciousness' ", Journal of Macromarketing, vol. 32, n³, pp. 266-279.

TEDIndia, (2009). «Shashi Tharoor : Why nations should pursue soft power », novembre. Consulté sur Internet (http://www.ted.com/talks/shashi_tharoor) le 30 juin 2015.

Teo, Stephen (2010). « Film and globalisation : From Hollywood to Bollywood », dans Bryan S. Turner (dir.), The Routledge International Handbook of Globalisation Studies, Londres, Routledge, pp. 413-428.

Thussu, Daya Kishan (2008). "The Globalization of 'Bollywood' : the Hype and the Hope », dans Anandam Kavoori, Aswin Punathambekar (sous la direction de), Global Bollywood, New York, London, New York University Press, pp. 97-115. 
Thussu, Daya Kishan (2012). « Bollywood globalisé », Théorème, n¹6, pp. 105-113.

Thussu, Daya Kishan (2013). Communicating India's soft power, Basingstoke, Palgrave Macmillan, 227 pages.

Trumpbour, John (2008). « Hollywood and the World : Export or Die », dans Paul Macdonald, Janet Wasko (sous la direction de), The contemporary film industry, Oxford, Blackwell Publishing, pp. 209-219.

Tunstall, Jeremy (2007). The media were American : US mass media in decline, New York, Oxford University Press, 480 pages.

Tyrrell, Heather (1999). « Bollywood versus Hollywood : Battle of the Dream Factories », dans Tracey Skelton et Tim Allen (sous la direction de), Culture and global change, London, New York,Routledge, pp. 312-319.

UNESCO, (2004). Avant-projet de Convention sur la protection de la diversité des contenus culturels et des expressions artistiques : Partie II : Commentaires spécifiques des États-membres, CLT/CPD/2004/ CONF.607/1, décembre.

UNESCO, (2006). Trends in Audiovisual Markets. Regional Perspectives from the South, Paris, UNESCO. Consulté sur Internet (http://unesdoc.unesco.org/images/0014/001461/146192e.pdf) le 10 juin 2015.

UNESCO Institute for Statistics, (2013). « Emerging Markets and the Digitalization of the Film Industry : An analysis of the 2012 UIS International Survey of Feature Film Statistics ", Information Paper, $\mathrm{n}^{\circ} 14$, août. Consulté sur Internet (http://www.uis.unesco.org/culture/ Documents/IP14-2013-cinema-survey-analysis-en.pdf) le 20 juin 2015.

UNESCO, (2015). Rapports périodiques quadriennaux : nouveaux rapports et résumé analytique, Paris, UNESCO. Consulté sur Internet (http://en.unesco.org/creativity/sites/creativity/ files/9igc_10_analysis_periodic_reports_fr.pdf) le 10 février 2016.

UNESCO-United Nations Development Program, (2013). Creative Economy Report 2013, New York, Paris, UNESCO/UNDP.

United States International Trade Commission, (2014). Recent Trends in US Services Trade, 2014 Annual Report, Washington, Publication Number : 4463. Consulté sur Internet (http:// www.usitc.gov/publications/332/pub4463.pdf) le 20 juin 2015.

Velayutham, Selvaraj (2008). «Introduction : the cultural history and politics of South Indian Tamil cinema », dans Selvaraj Velayutham (sous la direction de), The cultural politics of India's other film industry, London, Routledge, pp. 1-15.

Vlassis, Antonios (2015a). « Soft power, global governance of cultural industries and rising powers : the case of China », International Journal of Cultural Policy, Janvier, En ligne, DOI : 10.1080/10286632.2014.1002487.

Vlassis, Antonios (2015b). Gouvernance mondiale et culture : de l'exception à la diversité, Liège, Presses universitaires de Liège, 325 pages.

Vlassis, Antonios (2014). « Cultural development and technical and financial assistance on the basis of the CDCE » dans Lilian Richieri Hanania (sous la direction de), Cultural diversity in international law : Effectiveness and normativity of the 2005 Convention on diversity of cultural expressions, London, Routledge, pp. 167-180. 
Vlassis, Antonios (2011). «La mise en œuvre de la Convention sur la diversité des expressions culturelles : portée et enjeux de l'interface 'commerce-culture' ", Études internationales, vol. 42, $\mathrm{n}^{\circ}$ 4, pp. 493-510.

Vlassis, Antonios et Lilian Richieri Hanania (2014). « Effects of the Convention on trade negotiations ", dans Lilian Richieri Hanania (sous la direction de), Cultural diversity in international law : Effectiveness and normativity of the 2005 Convention on diversity of cultural expressions, London, Routledge, pp. 25-39.

Wagner, Christian (2010). «India's Soft Power : Prospects and Limitations », India Quarterly: A Journal of International Affairs, vol. 66, nº, pp. 333-342.

Wolfers, Anthony (1962). Discord and Collaboration. Essays in International Politics, Baltimore, John Hopkins University, 304 pages.

\section{NOTES}

1. Le Rapport sur l'économie créative 2013 des Nations unies souligne que le commerce mondial de biens et services créatifs est l'un des secteurs les plus dynamiques de l'économie mondiale. Il a plus que doublé entre 2002 et 2011, s'élevant au record de 624 milliards USD en 2011. Parallèlement, les pays en développement connaissent une croissance annuelle moyenne de 12,1 $\%$ pour l'exportation de biens créatifs (UNESCO-United Nations Development Program, 2013).

2. Rappelons qu'Anthony Wolfers a déjà abordé la question des ressources intangibles de la puissance, distinguant influence et pouvoir : la première est la capacité d'un acteur collectif à modifier le comportement des autres, à leur imposer sa propre volonté par la négociation, la diplomatie ou la cooptation ; le second use du recours à la menace ou à la force pour parvenir aux mêmes fins. En effet, la coercition est exclue au sein d'une relation d'influence (Wolfers, 1962 : 81-102). De son côté, dès 1948, Hans Morgenthau a décrit l'importance symbolique de la diplomatie culturelle : «if one could imagine the culture and the political ideology, with all its concrete imperialistic objectives, of state A conquering the minds of all of citizens determining the policies of state B, state A would have won a more complete victory and would have founded its supremacy on more stable grounds than any military or economic conqueror " (cité dans Pahlavi, 2013 : 567).

3. Pour un regard critique sur le soft power et les connotations normatives du concept, voir (Bohas, 2006 ; Chavagneux, 2010 : 26-29).

4. Avant les années 1990 , le gouvernement imposait un quota quantitatif d'importation de 100 films étrangers. En 2010, le nombre de films importés s'élève à 298 et en 2011 à 244.

5. Le 'B' se réfère à la ville de Bombay, actuellement connu comme Mumbai, qui est le cœur du cinéma commercial en langue hindi.

6. En 2003, l'industrie cinématographique indienne compte plus de 6000 producteurs (UNESCO, $2006: 311)$

7. Comme le note Joël Farges, «ceux qui osent affronter la réalité et raconter des histoires réelles, on les insulte ; on les censure, ou pire. Salaam Bombay de Mirna Nair et Fire de Deepta Mehta, deux films de femmes, ont tenté de raconter le vrai, de l'affronter, tout en essayant de rester du cinéma populaire. Le premier fut un succès international, mais un échec en Inde ; l'auteur du second reçut des menaces de mort, et des salles qui le programmaient furent incendiées » (Farges, 2000 : 166).

8. Le producteur est la personne physique ou morale qui prend l'initiative et la responsabilité de la réalisation de l'œuvre cinématographique. Le film terminé, il faut le présenter aux spectateurs. 
C'est le rôle du distributeur qui assure la mise en place du produit film : marketing, publicité, établissement des contrats avec des salles, organisation du passage d'un support à l'autre, etc.

9. Prem Ratan Dhan Payo (2015), Dhoom (2013), Bang Bang! (2014), Happy New Year (2014), Kick (2014).

10. Soulignons que l'Égypte, pourvue d'une industrie cinématographique dynamique, impose un quota quantitatif fixe pour des films indiens, alors que des quotas similaires ne sont pas imposés sur les films hollywoodiens.

11. Il s'agit des prix majeurs des festivals internationaux, tels que Palme d'or (Cannes), Lion d'or (Venise), Ours d'or (Berlin), Léopard d'or (Locarno), Coquille d'or (San Sebastian), ainsi que les Grands prix des jurys des festivals.

12. Nous visons à analyser la stratégie et les intérêts de l'Inde concernant l'interface 'commerceculture' et la coopération culturelle internationale. Le régime international de l'audiovisuel est d'ailleurs composé d'autres instruments multilatéraux se penchant sur la régulation internationale de la propriété intellectuelle, tels que le Traité sur le droit d'auteur et le Traité de Beijing sur les interprétations et exécutions audiovisuelles de l'Organisation mondiale de la propriété intellectuelle (OMPI), que l'Inde n'a pas encore ratifiés, ainsi que l'Accord sur les aspects des droits de propriété intellectuelle qui touchent au commerce de l'OMC. En revanche, vu une population nombreuse d'aveugles, l'Inde est le premier pays qui a ratifié en juin 2014 le traité de Marrakech de l'OMPI visant à faciliter l'accès des aveugles, des déficients visuels et des personnes ayant d'autres difficultés de lecture des textes imprimés aux œuvres publiées.

13. République d'Afrique centrale, République Dominicaine, Salvador, République de Gambie, Hong Kong, Inde, Israël, Japon, Kenya, Corée du Sud, Lesotho, Malaisie, Mexique, NouvelleZélande, Nicaragua, Singapore, Thaillande, États-Unis.

14. Notons que le Mexique et le Brésil sont des exportateurs importants de telenovelas à un grand nombre de pays d'Amérique latine. De leur côté, Hong Kong, le Japon, Taiwan et l'Inde ont des intérêts d'exportation des services audiovisuels dans plusieurs pays d'Asie, comme aussi l'Égypte vers le Moyen-Orient et l'Afrique.

15. Selon l'OMC, les services audiovisuels incluent des services de production et de distribution de films cinématographiques et de bandes vidéo, des services de projection de films cinématographiques, des services de radio et de télévision, des services de diffusion radiophonique et télévisuelle et des services d'enregistrement sonore.

16. Entretien auprès d'un haut fonctionnaire de l'OMC, 8 avril 2014.

17. Source : Mojo, URL : http://boxofficemojo.com/alltime/world/.

\section{RÉSUMÉS}

Cet article s'interroge sur la place de l'industrie cinématographique de l'Inde dans le renforcement de son soft power et sur quelle mesure celle-ci permet à l'Inde de construire un statut de puissance culturelle active. En ce sens, il s'agit d'abord d'analyser les caractéristiques institutionnelles du système cinématographique indien, avant de se pencher sur le rôle de l'Inde au sein du régime international de l'audiovisuel, et enfin de terminer sur la concurrence culturelle mondiale à laquelle l'Inde est confrontée. Il s'avèrera que les structures domestiques sont un facteur majeur en vue de saisir la volonté d'influence de l'Inde sur la scène internationale et sa place dans l'économie audiovisuelle mondiale au sein de laquelle Hollywood dispose encore de capacités de financement sans égal et de stratégies de distribution mondiales. 
This article deals with the importance of the Indian film industry for the strengthening of India's soft power and with the capability of the country to build an international status of active cultural power. As such, on the one hand, I seek to analyze the institutional features of the Indian movie system and to focus on the role of India within the international regime for audiovisual sector, and on the other hand, I aim to emphasize the worldwide cultural competition that India faces. The findings will show that the domestic structures are a major factor in order to understand the influence of India and its status within the world audiovisual economy, within which Hollywood has highly financing abilities and global distribution strategies.

\section{INDEX}

Keywords : Hollywood, India, movie industry, soft power, UNESCO, World Trade Organization

Mots-clés : cinéma, Hollywood, Inde, Organisation mondiale du commerce, soft power, UNESCO

\section{AUTEUR}

\section{ANTONIOS VLASSIS}

Chargé de recherches et chargé de cours, Fonds national de la recherche scientifique (FNRS)-

Center for International Relations Studies (CEFIR), Université de Liège, Belgique

antonios.vlassis@gmail.com 